\title{
VARIABLE SELECTION FOR ROAD SEGMENTATION IN AERIAL IMAGES
}

\author{
Sven Warnke, Dimitri Bulatov \\ Fraunhofer IOSB, Ettlingen, Germany, \{name.surname\}@iosb.fraunhofer.de
}

Commission II, WG II/4

KEY WORDS: Classification, Feature Selection, Logistic Regression, Random Forests, Road Extraction

\begin{abstract}
:
For extraction of road pixels from combined image and elevation data, Wegner et al. (2015) proposed classification of superpixels into road and non-road, after which a refinement of the classification results using minimum cost paths and non-local optimization methods took place. We believed that the variable set used for classification was to a certain extent suboptimal, because many variables were redundant while several features known as useful in Photogrammetry and Remote Sensing are missed. This motivated us to implement a variable selection approach which builds a model for classification using portions of training data and subsets of features, evaluates this model, updates the feature set, and terminates when a stopping criterion is satisfied. The choice of classifier is flexible; however, we tested the approach with Logistic Regression and Random Forests, and taylored the evaluation module to the chosen classifier. To guarantee a fair comparison, we kept the segment-based approach and most of the variables from the related work, but we extended them by additional, mostly higher-level features. Applying these superior features, removing the redundant ones, as well as using more accurately acquired 3D data allowed to keep stable or even to reduce the misclassification error in a challenging dataset.
\end{abstract}

\section{INTRODUCTION}

Motivation Extracting roads from remote sensing images is important in a number of different applications, for example traffic management, city planning, road monitoring, GPS navigation and map updating (Wang et al., 2016). However, until now, no fully automated road network detection method is applied in praxis, see Rottensteiner et al. (2013). The reason lies in the difficulty of the classification problem. There are many factors that make road network extraction from remote sensing images challenging. First of all, the dependence on the sensors and resolution cause a variety of problems; moreover, even if same sensors and resolutions are used, appearance of roads in remote sensing images can vary wildly. Some reasons are weather and illumination effects and, very importantly, shadows and occlusions caused by high buildings, tree crowns, moving or parking vehicles and tiles of rubbish in developing countries. Because buildings and vehicles are indispensable part of urban terrain, road extraction from urban scenes is considered even more difficult than in rural areas (Hu et al., 2014).

Previous work The challenges mentioned above demonstrate that probably any conceivable rule for road pixel extraction from 2D or 3D information has a large number fo counterexamples, both false positive and false negative. One could think about thresholding relative elevations (also knows as normalized Digital Surface Model, nDSM) over the ground; Here, bridges are examples of false negatives and farmlands represent examples of false positives, leaving aside the fact that extraction of ground pixels is not always trivial and widely error-free. Thus, modern approaches use combination of rules that support each other on their way towards a reliable road pixel extraction. Thinkable rules are nDSM, Normal Difference Vegetation Index (NDVI), color information from different color spaces (Wegner et al., 2015), lines detected in images (Ünsalan and Sirmacek, 2012), stripes detected in nDSM for representing street canyons (Hinz, 2004), surface roughness ( $\mathrm{Hu}$ et al., 2014), spatial signature measure employed by Jin and Davis (2005), filters, descriptors and textons employed by Montoya-Zegarra et al. (2015); Poullis and You (2010); Wegner et al. (2015), methods based on morphological profiles (Valero et al., 2010) up to the approaches based on Convolutional Neural Networks (Sherrah, 2016), where the problem is solved by applying very large numbers of features (neurons) and huge amounts of training data.

The variety of rules in this fragment of the seemingly nearly endless list of ways to extract road pixel confirm the non-triviality of the task. However, one can identify tendencies towards a large number of generic features without explicit semantic meaning. Also, it can be followed that a combined evaluation of features, in particular extracted both in two- and three-dimensional data, is more promising than relying on just one source of information. Accurate, high-resolution 3D information can be extracted by means of a stereo-matching procedure, such as Lemaire (2008).

The features presented above have in common that they rely on local properties of a structural image element (pixels, superpixels,...). The considered neighborhood of a pixel can be quite large (such as receptive fields in a convolutional neuronal network), but for classification tasks, it is limited. However, a particularly important property of the road network is its connectedness. Several excellent contributions exist which aim at extraction of (curvy)linear structures by fitting low-cost paths between the seeds, which are pixels with locally minimum values of the cost function, such as tubular measure (Türetken et al., 2012). For combined evaluation of image and elevation data, Wegner et al. (2015) proposed a pioneering approach for extraction of road superpixels. In the first step of this approach, over-segmentation of the image material into homogeneous regions called superpixels (Veksler et al., 2010) is created. Second, features from images and elevation maps are extracted segment-wise and classified by a standard algorithm, such as random forests (Breiman, 2001). In the third step, minimum-cost paths are calculated between points with high probability of being roads. In the last fourth step, a conditional random field is applied for smoothing, 
using the minimum cost paths as higher order cliques. The superpixels along the reliable paths are thus encouraged to belong to the road class. The latter two steps of the method can be interpreted as post-processing of the classification result obtained by random forests. Obviously, the quality of the final road network extraction depends to a great part on the accuracy of this preliminary classification.

Bottleneck: Variable selection Clearly, the main focus of the contribution of Wegner et al. (2015) lay on minimum cost paths. However, we feel that the step of preliminary classification did not receive the attention and care its importance deserves. Especially, in the list of used variables, some of them were quite obviously redundant and many useful variables missed. Missed are those higher-level features that allow to combine spectral channels and elevation data and thus may perform better in shadow regions. Redundant features are known to have only marginal detrimental effect on random forests (Genuer et al., 2010), but a dramatic degradation can result for other classifiers, such as logistic regression (Cox, 1958). As a consequence, any method, including the approach of Wegner et al. (2015), could benefit greatly - both with respect to the performance and the computing time - from a careful selection of variables (features).

Methods for automatic variable selection are being applied in basically all machine-learning areas. The dramatically increased amount of data and features - as highlighted in our previous considerations - commonly used in modern machine learning due to the growing capabilities of sensors and computers, underlines the importance of automatic feature selection in basically all areas of machine-learning. Additionally, in many applications, expert knowledge is not always available or sufficient to select variables.

Since for a set of $p$ variables, the number of possible subsets is $2^{p}$, the problem of variable selection is known to be NP-hard. Hence, no feature selection method that terminates in a reasonable amount of time performs optimally in all situations (Kohavi and John, 1997; Bolón-Canedo et al., 2015). It is, therefore, not surprising that an immense and ever growing number of different heuristic strategies exist (Bolón-Canedo et al., 2015; Stańczyk and Jain, 2015), so many in fact that it makes choosing one of them difficult since it requires a relatively deep understanding not only of the mechanism of available feature selection methods, but also and in particular, the functionality of the underlying classifier.

Contribution In this work, we adopted from the superpixelwise classification framework and the features generated from filter banks Wegner et al. (2015); that is, we omitted the postprocessing steps in order not to gloss over the raw classification results. However, we extend the variable set of Wegner et al. (2015) by our variables and performed classification with two methods. On the one hand, we applied the random forest classifier, which is a conceptually simple, but powerful tool whose mathematical properties are however not sufficiently explored yet. On the other hand, we employed logistic regression. Although its application is not recommendable if the data cannot be approximately separated into classes by hyper-planes or if variables are strongly correlated, this is actually the reason why we are interested to measure its performance while testing our (sometimes highly redundant) variables. Besides, logistic regression is mathematically well understood due to its inclusion into the class of generalized linear models for which properties asymptotic in terms of training data can be proved (Fahrmeir and Kaufmann, 1985; Warnke, 2017).
Inspired by the work of Kohavi and John (1997), we implemented for both classifiers a wrapper framework, which allows computation and evaluation of models with part of the training data and feature subsets. Stepwise forward and backward selection presuppose extending or reducing these subsets until some stopping criterion is satisfied. Thus, our main contribution is made up of a simple, yet powerful, method of feature selection with a rather intuitive mechanisms. Furthermore, as all wrappers, it directly links selection to prediction accuracy, a measure whose utility is obvious and which can be tracked back to the visited feature subsets and their evaluation result. For random forest, this is outof-bag misclassification rate, and for logistic regression, it is the Wald-test. Both are built into their respective models; as a consequence, most users will be familiar with them, which keeps the selection process easily understandable.

We applied and evaluated different variable selection methods to road extraction from the Vaihingen dataset (Rottensteiner et al., 2013), an often used benchmark for urban classification from remote sensing. The learning algorithms we used are random forests, as proposed by Wegner et al. (2015), but also logistic regression.

Organization The paper is structured as follows. In Sec. 2, we give a description of our wrapper and its application for our classifiers. Note that we will skip the description of these classifiers since these are standard, established methods; the interested reader can find their detailed description and critical juxtaposition with respect to feature selection in Warnke (2017). Next, we discuss in Sec. 3 the relevant features. In Sec. 4, we present our results: Feature sets selected by each classifier and each selection tool as well as their performance. Sec. 5 summarizes the contents of this work and outlines several ideas of future research.

\section{WRAPPER METHOD FOR VARIABLE SELECTION}

There are three general classes of variable selection algorithms. First, filter methods basically presuppose assessing each variable's usefulness through the training data only and discard the less relevant variables. Thus, they do not depend on the underlying classifier. Second, methods embedded into the model building process penalize the models that are too complex, thus allowing to establish which variables best contribute to the model accuracy and which are superfluous. The third category is referred to as wrapper methods. These are recursive procedures which comprise: Model inference based on a subset of variables, evaluation of the model using e. g. $n$-fold cross validation and updating the subset according to the result of the evaluation. The procedure terminates if no better subset was found within the last couple of steps of the search algorithm. Because wrapper methods connect variable selection to prediction accuracy of the underlying classifier, they are particularly interesting to the authors.

To summarize, wrapper methods can normally be divided into the following parts, covered in the rest of this section: Starting set, search algorithm, stopping criterion, and evaluation method. In the following, we will discuss these parts in more detail.

The starting set of a wrapper method is usually either the empty or the full set of features. In the first case, the quintessence of the search algorithm is to add, successively, variables (individually or multiples at the same time) to the starting set. This category of methods is commonly denoted as forward selection. The motivation to add not single but promising combined sets of variables 
comes from the fact that in the worst case, adding single features requires evaluating $O\left(p^{2}\right)$ models, where $p$ is the number of features, which may be very high. To avoid this, depth-first search is simulated. In the second case, known as backward selection, variables are gradually removed from the current set. Because creation of the models containing fewer predictors is less computationally expensive, forward selection is often preferred in praxis due to its greater speed. However, for backward selection, usually, less models have to be instantiated.

As for the stopping criterion, an intuitive way to do this would be stopping as soon as adding a further feature does not improve the accuracy of prediction. However, an extreme example, the well-known XOR-problem, shows that this strategy is suboptimal and that care should be taken to prevent the algorithm ending up in a local optimum. In the XOR-problem, none of two variables improves the performance, but their combination does. The necessary condition for selecting both relevant variables within a stepwise forward selection algorithm is to let the search terminate if in at least the last two steps no improvement has been achieved. Note that this condition is not sufficient, because one of two features must make it into model up to this point, in order to reach the second feature in the next iteration. It is, however, unlikely if we assume that any of two features has no merit on its own.

The remaining question of evaluation method can always be solved using the $n$-fold cross-validation. It is considered a very reliable way to estimate prediction accuracy of a model (Kohavi and John, 1997), but it is however expensive since $n$ models have to be fitted. Because of this and because of a rather undesired invariance of this method with respect to the underlying classifier, we are interested to design a classifier-internal evaluation strategy. For classifiers selected for this work, namely logistic regression and random forests, the respecting evaluation methods will be reviewed in both concluding paragraphs of this section.

For logistic regression, it is possible to refer to the so called Waldstatistics, which allows to detect which variables of the current set are redundant; during the backward selection, it can be applied either to the current set of features at each iteration, striving to remove one variable, or to the whole set of features if order to filter out the apparently less useful and redundant features. From the asymptotic properties of logistic regression, it is known that the sought parameter vector $\beta$ is normally distributed

$$
\hat{\beta} \sim \mathcal{N}\left(\beta_{0}, F(\hat{\beta})^{-1}\right),
$$

where $\beta_{0}$ is the expectancy and $F$ the Fisher information defined as the inverse covariance matrix of the gradient of the loglikelihood function with respect to $\beta$. Using the approximate normality of $\beta$, the Wald-test is supposed to test the hypothesis $H_{0}: \beta_{q+1}=\cdots=\beta_{p}=0$, for some $q, 0 \leq q<p$, which would mean that the variables corresponding to the last $p-q$ entries of $\beta, \beta_{q+1}, \ldots, \beta_{p}$ are not needed for our model. To reject the hypothesis $H_{0}$, it can be followed that the Wald-statistics

$$
W=\tilde{\beta}^{T} \tilde{F} \tilde{\beta}\left(\sim \chi_{p-q}^{2}\right)>\chi_{p-q}^{2}(0.95),
$$

where $\tilde{\beta}=\left[\beta_{q+1}, \ldots, \beta_{p}\right]^{T}, \tilde{F}$ is the lower $(p-q) \times(p-q)$ submatrix of $F$, and $\chi^{2}$ is the well-known Chi-squared distribution. Note that (2) holds if at least one of the entries of $\tilde{\beta}$ deviates from zero and thus, one of the corresponding variables is needed for our model. Of course, in praxis, the test is often used the other way round. A set of predictors is removed from the model if their Wald-statistic is not significant at a predefined level, opting thus for simpler models once it could not be shown that all predictors of a more complicated one are needed.

For random forests, instead of costly cross-validation, the easily available out-of-bag prediction measure makes possible a much faster estimation of the model prediction accuracy. For a random forest $T$, the rate of Out-of-Bag misclassification is defined as:

$$
E_{\text {ООВ }}(T)=\frac{1}{\# X} \#\left\{(x, y) \in X \mid T_{\text {ООВ }}(x, y) \neq y\right\} .
$$

where $X$ is the set of training samples $(x, y)$ and $x$ and $y$ are the features and the labels, respectively, while \# denotes the cardinality of a set. Further, $T_{O O B}(x, y)$ denotes the Out-of-Bag prediction for training sample $(x, y)$, i.e. the class that is predicted by the majority of trees within $T$ where $(x, y)$ was out-of-bag. There is empirical evidence that the accuracy of the Out-of-Bag misclassification rate is almost the same as if one would apply an additional test-set of the same size (Breiman, 1996).

\section{EXTRACTED FEATURES}

Since the number of features that could possibly be extracted is seemingly endless, this step is always a compromise. On the one hand, we wish to incorporate all the useful information contained in the image material, since due to our subsequent feature selection, we can accept taking in a lot of irrelevant or redundant variables in order to show the capabilities of variable selection. On the other hand, however, too many useless features can become prohibitively expensive for the wrapper methods with respect to computing time. Nevertheless, we align ourselves to the choice of Wegner et al. (2015), extend it by several new features that are popular in photogrammetry and remote sensing, and wish to demonstrate that even after a careful, knowledge-based extraction of features, automatic variable selection is highly beneficial.

A common approach in computer vision is convolving the image material with a number of different filters and taking the resulting filter responses as features (Cula and Dana, 2004; Varma and Zisserman, 2005; Winn et al., 2005). The applied filters usually detect edges or smoothen the image. For example, Wegner et al. (2015) applied the filter bank of Winn et al. (2005) to each of the three channels of an image, transferred into the opponent Gaussian color space (OGC space) (Geusebroek et al., 2001) as well as the relative elevation value. The mean value and standard deviation of all these features over all pixels of a segment were then the output of their feature extraction procedure. Hence, in total, there were:

$\{3 \times 3$ [Gaussians for every channel] $+4 \times 2$ [Filter kinds for the first channel] +1 [rel. elevation] $\} \times 2$ [mean and standard deviation $]=36$ variables. It seems that some of these predictors are redundant and that some measures (like NDVI, Normal Vegetation Differential Index), commonly used in classification for remote sensing applications, are not taken into account. For our work, this was the main motivation.

We used the MR8 filter bank of Varma and Zisserman (2005) instead of the filter bank of Winn et al., since it led to a higher accuracy. The main reason for this is that it incorporates more edge and ridge detection, which is essential for texture analysis. Also, it is invariant with respect to the image rotations. Besides, in segment-wise classification, applying Gaussians and Laplacians with different parameters does not usually provide more information since the subsequent averaging over the superpixel results in basically identical features. Last but not least, Burghouts and 
Geusebroek (2009) claim that the filters of Varma and Zisserman (2005) are better suited for application to images in the OGC space while the filter set of Winn et al. (2005) is designed for the Lab color space.

Moreover, we collected unfiltered channels of the orthophoto and nDSM in order to take into account different filter sizes from the Winn bank of filters, preferred by Wegner et al. (2015). We refer to the total of 56 variables:

$\{8$ [filters in MR8 filter bank] $\times 3$ [channels] +3 [unfiltered channels] +1 [rel. elevation] $\} \times 2=56$ as Wegner-like. Additionally, we have 22 more variables. The first 16 of them arise from the fact that we also subject the nDSM to the MR8-based filtering (note that as always we store average and standard deviation of every segment), and only image channels. The DSMs stem from a very accurate multi-baseline approach. As a consequence, also the features derived from the DSM (planarity, scattering etc.) are highly discriminative and almost comparable with those extractable from laser point clouds.

The remaining six features use combinations of channels. First, the well-known NDVI (also here, average and standard deviation were taken). Second, stripes computed in the nDSM and in the orthophoto were calculated. These stripes are formed from pairs of nearly parallel straight lines detected in the image. For application of stripes for detecting man-made structures, we refer to Soergel et al. (2006), from where we also took the technical details for the computation of stripes. Stripes are detected in the $\mathrm{nDSM}$ and in the intensity image of the orthophoto, thus yielding four more variables. In the following, we summarize the differences between our variables and those of Wegner et al. (2015).

1. Unfiltered channels,

2. Filter bank: MR8 instead of Winn-filters $\rightarrow$ Wegner-like features,

3. Application of MR8 filter bank to nDSM,

4. NDVI- and stripes-based features.

Classification For training with a certain training set using a specific feature set, the output model is the set of decision trees in case of random forests classifier and the parameter vector $\hat{\beta}$ for logistic regression. For logistic regression, the probability $P$ of a superpixel to belong to the road class is given by the logistic function

$$
P=\frac{\exp \left(\hat{\beta}^{T} x\right)}{1+\exp \left(\hat{\beta}^{T} x\right)}
$$

where $x$ is the feature vector. For random forests, $P$ is the percentage of trees which output it as road class (we utilized 200 trees in our experiments). The local classification result is obtained by assigning the superpixels the road class if $P>0.5$ and non-road otherwise. Wegner et al. (2015) used $P$ for their non-local optimization post-processing step.

For convenience, we summarize below our three-step algorithm:

1. Perform variable selection on a part of the training data which yields a feature subset

2. Induce a model using these features and the complete training data

3. Output the probability of the test data.

\section{RESULTS}

The considered dataset is the publicly available ISPRS benchmark Vaihingen (a town in Southern Germany) for urban area object classification, see Rottensteiner et al. (2013), provided by the German Society for Photogrammetry, Remote Sensing and Geoinformation (DGPF), Cramer (2010). In total, there are 16 patches containing the images at a resolution $\approx 0.1 \mathrm{~m}$, the corresponding DSMs, created by the method of Lemaire (2008), as well as the labeled ground truth, created by SIRADEL corporation (www.siradel.com). In the images, the near infrared is present instead of the blue channel. For a better overview, we want to outline in Sec. 4.1 important remarks on data preparation as well as the evaluation criteria while in Sec. 4.2, evaluation results will be presented.

\subsection{General remarks on preparation of the data}

nDSM extraction and superpixel generation Firstly, since the relative elevatation is clearly a more suitable variable for a learning algorithm than the absolute height, we obtained for every patch the nDSM, . This is done by calculating the ground surface using the procedure of Bulatov et al. (2014) and subtracting it from the DSM. Secondly, segmentation into superpixels is carried out by means of the compact superpixels algorithm (Veksler et al., 2010). This algorithm uses for each superpixel a data term, which prevents it from growing outside of a predefined box, and a smoothness term penalizing the weighted number of pixels shared by two adjacent superpixels. Since this smoothness penalty is submodular, the alpha-expansion method (run over all superpixels and several iterations) with maximum flow (Boykov et al., 2001) as the core methods allows to obtain a strong local minimum of the resulting function. Next, we proposed a filtering procedure, since some segments are too small and do not contain enough information. They are fused with neighbors. In the segmentation we used throughout this work, the average number of pixels in a segment as about 440 and the number of segments thus was around 10 to 12 thousands which makes the test tractable (not more than several seconds).

Features computation Similarly to Wegner et al. (2015), we extracted the mean and the standard deviation for all features over segments. However, since the near-infrared replaces the blue channel, the transformation into OGC-space, originally designed for true RGB images, is, strictly speaking, invalid. Nevertheless, Wegner et al. (2015) converted the image material, treating them just as though they were RGB images without even a side note. Even though this seems questionable, our experience showed that calculating features from the transformed images achieved better accuracy than those extracted directly from the infrared, red and green values. Because of this, because of a consistent comparison, and because we are planning to evaluate our features rather than fine-tune them, we decided to retain this strategy.

Evaluation strategy The models we compare in this work use feature sets resulting from:

1. The standard forward selection,

2. the standard backward selection,

3. all variables discussed in Sec. 3,

4. all Wegner-like variables,

and both aforementioned classifiers. Furthermore, the questions we wish to find answer for are: 
1. How many variables each of our classifiers considers as necessary,

2. how the reduction of variables changes the prediction accuracy, in particular, with respect to the full set of variables and to the results of related work,

3. overall performance evaluation

4. finally, which are the trend in variables preferred by logistic regression and random forests.

To answer the first question, we successively used each of the segmented patches as test data while the remaining patches served as training data. This means that after variable selection, every of 78 features of Sec. 3 can at most be called 16 times. Thus, we can derive the average size of the feature sets produced by the feature selection methods. Analogously, we can store for all variables the number of patches it was needed in, to give answer to the last question. For the second question, we specified, according to Heipke et al. (1997), the standard measures of classification, such as completeness, correctness and quality. To compute these measures, we assessed, which segments lie in ground truth to more than or less than $50 \%$ in the street class to obtain true and false segments, respectively. From this as well as the positives and negatives of our classification result, the measures of Heipke et al. (1997) are derived using the well-known terms. For more thorough performance evaluation, for example, ROC curves, we refer to Warnke (2017), since otherwise we would explode the scope of this work. Thus, a brief reference to two classification examples will be provided to respond to the third question and a few concluding recommendations on the selection of features for both classifiers are given to provide an answer of the fourth question.

\subsection{Evaluation}

Reducing feature set We start analyzing the overall performance of the variable selection methods with respect to their ability of variable reduction. In Fig. 1 we see the number of variables remaining after running forward and backward selection for both logistic regression and random forests. It is evident that our goal was successfully achieved since feature sets are always reduced to some extent and significantly in three of the four methods. Especially, forward selection brought a considerable improvement: The average number of remaining variables was below 32 for logistic regression and even below 17 for random forests. However, results tend to lie close to the starting point of the search, i. e. the empty set for forward selection and the full set of predictors for backward elimination. It may seem worrying: Since forward and backward methods are supposedly optimizing the same target function and only differ in the way they search through the space of candidate subsets, one should expect more similar results. It seems that the search gets stuck in a local minimum after a relatively short time and that this tendency will aggravate with a higher number of features. It is worth mentioning that with respect to the backward selection, the situation is considerably better for logistic regression: Since logistic regression is more sensitive towards redundant variables, discarding them usually causes a noticeable improvement. In the case of random forests, redundant variables do not have such a negative impact on a models accuracy. Although this is a treasured property of random forests, for a wrapper-type feature selection, however, it becomes cumbersome, since it leads to premature termination of the search. The mentioned differences between forward and backward selection should be kept in mind when choosing a feature selection method. As a rule of thumb, forward selection methods are suitable for a drastic reduction of the variable set whereas backward methods focus on prediction accuracy.
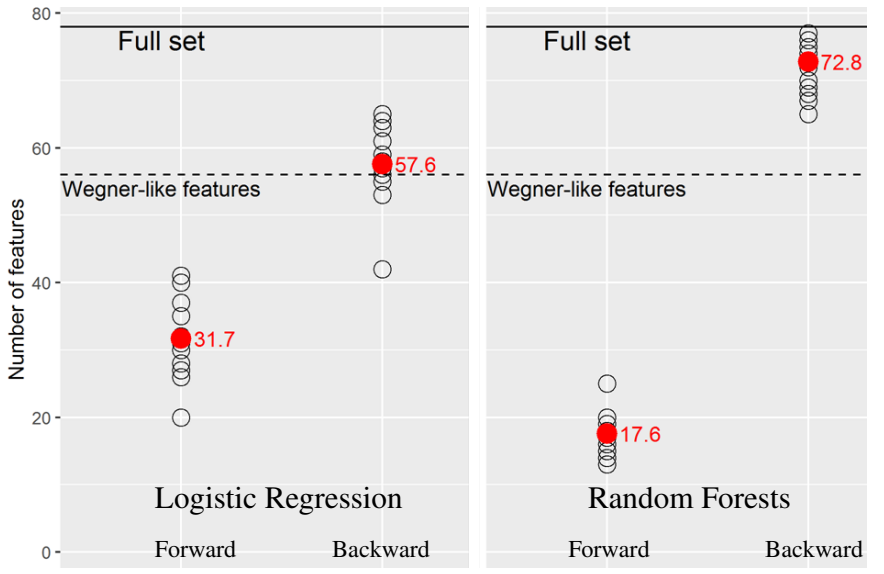

Figure 1: Cardinalities of the produced feature sets for different patches, indicated by black circles; the average cardinalities are depicted in red.

Performance of reduced sets Another good news is that, discarding even a significant amount of variables through variable selection does not affect the performance negatively. On contrary, as shown in Fig. 2, it becomes clear that the reduced set of features can improve the performance for Logistic Regression, while for Random Forests, it remains approximately equal (for the different sensitivity towards redundant variables as explained above). An important point to note is that the addition of features to the Wegner-like features, increased performance noticeably. To provide a direct comparison of our method with the actual results of Wegner et al. (2015), it must be kept in mind that the elevation information was computed by a state-of-the-art method and is of a superior quality to that used in their paper. Moreover, the measures of completeness, correctness, and quality of Heipke et al. (1997) depend highly on the discrimination threshold, which was not provided. Nevertheless, we found discrimination thresholds for both of our classification methods $(0.5$ for random forests and 0.65 for logistic regression) that result in improvements in all three of those values at the same time (see Table 1). Thus, the usefulness of our modifications to the method of Wegner et al. (2015), even after application of the post-processing steps, is shown.

From Table 1, we also gather that random forests outperformed logistic regression. Another point that speaks for the choice of random forests as a learning algorithm over logistic regression within the procedure of Wegner et al. (2015) is the nature of the produced a-posteriori probabilities: Logistic regression tends to output probabilities close to either zero or one, whereas random forests convey uncertainty better, thus making post-processing steps like the proposed minimum-cost paths more promising.

\begin{tabular}{|l|c|c|c|}
\hline Method & Quality & Comp-ness & Corr-ness \\
\hline Wegner's RFs & 0.65 & 0.77 & 0.81 \\
Wegner's paths & 0.68 & 0.81 & 0.81 \\
\hline Our LR & 0.692 & 0.817 & 0.819 \\
Our RF & 0.722 & 0.846 & 0.831 \\
\hline
\end{tabular}

Table 1: Comparison of the pixel-wise classification obtained with the results of Wegner et al. (2015, Table 1).

Overall performance evaluation The measures of pixel-wise classification in Table 1 and in Fig. 2 are suitable to assess the 

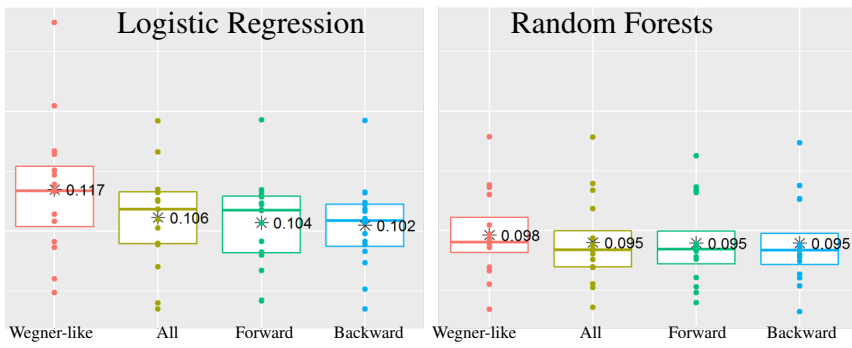

Figure 2: Resulting (superpixel-wise) average class error of the models trained on the feature sets produced by each variable selection method for each learning algorithm. Colored points denote the performance (percentages of falsely classified superpixels) of the relevant algorithm for the 16 patches. The colored lines and stars stand for average and median values, respectively.

performance of the method quantitatively. However, in Fig. 2, we noticed that the quality of our classification results varies greatly from patch to patch. Hence, our next goal is a qualitative assessment of the classification results, for which we refer to Fig. 4, p. 8. It is well-known that road extraction from remote sensing is difficult because of shadows (see Sec. 1), which are the most common reason for misclassification occurence in our results as well. The presence of dark shadows in an urban scene depends on the size of the constructions and trees. Beside, some parts of Vaihingen have many narrow alleys while others are dominated by broad streets. Here is also where the problem of under-segmentation comes into play. Finally, a huge problem for classification are rare types of ground cover. For instance, patch number 26 of the Vaihingen data set is the only one where a river appears. Since its appearance in color, texture, and height resembles impervious surface, it gets misclassified almost completely since the learning algorithms did not see water surface in the training data.

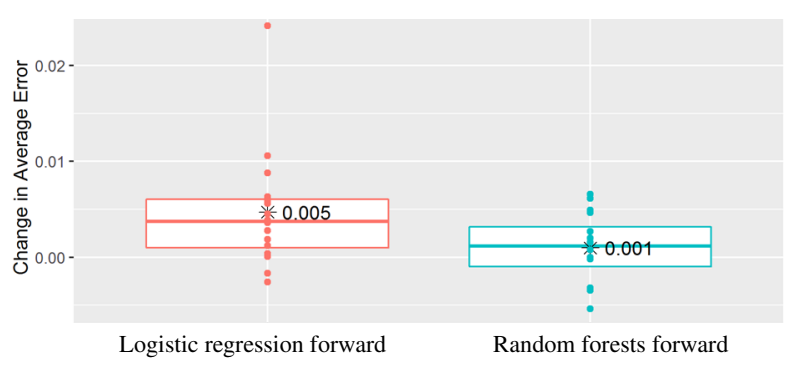

Figure 3: Resulting change in average class error if the models are trained on the variable sets produced for specifically for the other learning algorithm by forward selection. Colored points denote the performance (percentages of falsely classified superpixels) of the relevant algorithm for any of 16 single images. The colored lines and stars represent average and median values, respectively.

Trends of selected features As for variables chosen by both classifiers, Random Forests preferred the (segment-wise) averages to the standard deviations and filtered to the non-filtered features. In forward selection, actually almost all standard deviations were considered irrelevant, with exception of relative elevation and filter 1. From the average values, features based on the relative elevation were usually called slightly more often than those based on the three channels of the images. Logistic regression almost equally relies on averages values and standard deviations. It takes more variables resulting from the filter bank, especially the 7-th and 8-th filters. Features based on stripes and average of NDVI were selected very often both for logistic re- gression and for random forests. This supports our expectations about the importance of skillfully combining different channels and developing higher-level features that go beyond the borders of the segments and incorporate context information. For more details, we refer to Warnke (2017).

The feature sets produced by our wrapper-type feature selection methods are specific to the machine learning method for which they were conducted. It can be observed on the right part of Fig. 3 that on average, the set of variables recommended by the forward selection method of logistic regression results in a slightly worse classification accuracy by means of random forests than the feature set customized for this classifiers. On contrary, if we use the set of variables recommended by Random Forests for Logistic Regression as a classifier, the accuracy sinks considerably. The fact that the relevance of different features depends on the machine learning algorithm may appear surprising at the first glance. However, since the mechanisms of these algorithm differ substantially, these are also different (aspects of) variables that come into play. This demonstrates, on the one hand, the theoretical strength of wrappers, since they choose variables that are useful for a particular learning algorithm. On the other hands, it helps to emphasize the importance of easy-to-use variable selection methods that allow every user selecting those variables that are specifically useful for his/her application and classification method.

\section{CONCLUSIONS AND OUTLOOK}

We investigated the merit of two simple wrapper approaches for variable selection and tested them extensively with two classifiers, several dozens of features and an extensive amount of training and test data. From our experiments, we saw that there is a lot of potential for optimizing the classification quality by choosing appropriate variables and performing variable selection both with respect to accuracy and costs. The features we added to those mentioned in Wegner et al. (2015), such as DSM filters, vegetation index and presence of stripes, allowed to achieve a higher accuracy. However, this improved accuracy does not necessarily come at the cost of a bigger feature space. As we have seen, it is possible to reduce the extended feature space, even below the size it had before our features were added, while keeping the accuracy stable or even improving it.

We could see that the relevance of different features depends on the machine learning algorithm. Thus, we demonstrate the theoretical strength of wrappers, since they choose features that are useful for a particular machine learning algorithm, contrary to filtering methods, which assess suitability of every feature. However, the fundamental differences between the variable sets produced by forward and backward selection should be noted. Concerning the choice of classifier, random forests are preferable over logistic regression because they produce a higher classification accuracy. Moreover, as our first experiments showed, they are more suitable for the minimum cost paths employed by Wegner et al. (2015), since they better convey uncertainty which simplifies post-processing the results. However, it remains to inspect whether other classifiers like neural networks or support vector machines might lead to even better classification results.

\section{References}

Bolón-Canedo, V., Sánchez-Maroño, N. and Alonso-Betanzos, A., 2015. Feature selection for high-dimensional data. Springer. 
Boykov, Y., Veksler, O. and Zabih, R., 2001. Fast approximate energy minimization via graph cuts. IEEE Transactions on pattern analysis and machine intelligence 23(11), pp. 12221239.

Breiman, L., 1996. Out-of-bag estimation. Technical report, Citeseer.

Breiman, L., 2001. Random forests. Machine learning 45(1), pp. 5-32.

Bulatov, D., Häufel, G., Meidow, J., Pohl, M., Solbrig, P. and Wernerus, P., 2014. Context-based automatic reconstruction and texturing of 3D urban terrain for quick-response tasks. ISPRS Journal of Photogrammetry and Remote Sensing 93, pp. $157-170$.

Burghouts, G. J. and Geusebroek, J.-M., 2009. Material-specific adaptation of color invariant features. Pattern Recognition Letters 30(3), pp. 306-313.

Cox, D. R., 1958. The regression analysis of binary sequences. Journal of the Royal Statistical Society. Series B (Methodological) pp. 215-242.

Cramer, M., 2010. The DGPF test on digital aerial camera evaluation - overview and test design. Photogrammetrie, Fernerkundung, Geoinformation 2, pp. 73-82.

Cula, O. G. and Dana, K. J., 2004. 3d texture recognition using bidirectional feature histograms. International Journal of Computer Vision 59(1), pp. 33-60.

Fahrmeir, L. and Kaufmann, H., 1985. Consistency and asymptotic normality of the maximum likelihood estimator in generalized linear models. The Annals of Statistics 13(1), pp. 342368.

Genuer, R., Poggi, J.-M. and Tuleau-Malot, C., 2010. Variable selection using random forests. Pattern Recognition Letters 31(14), pp. 2225-2236.

Geusebroek, J.-M., Van den Boomgaard, R., Smeulders, A. W. M. and Geerts, H., 2001. Color invariance. IEEE Transactions on Pattern Analysis and Machine Intelligence 23(12), pp. 13381350.

Heipke, C., Mayer, H., Wiedemann, C. and Jamet, O., 1997. Evaluation of automatic road extraction. International Archives of Photogrammetry and Remote Sensing 32(3 SECT 4W2), pp. 151-160.

Hinz, S., 2004. Automatic road extraction in urban scenes and beyond. International Archives of Photogrammetry and Remote Sensing 35(B3), pp. 349-354.

Hu, X., Li, Y., Shan, J., Zhang, J. and Zhang, Y., 2014. Road centerline extraction in complex urban scenes from lidar data based on multiple features. IEEE Transactions on Geoscience and Remote Sensing 52(11), pp. 7448-7456.

Jin, X. and Davis, C. H., 2005. An integrated system for automatic road mapping from high-resolution multi-spectral satellite imagery by information fusion. Information Fusion 6(4), pp. 257-273.

Kohavi, R. and John, G. H., 1997. Wrappers for feature subset selection. Artificial intelligence 97(1), pp. 273-324.

Lemaire, C., 2008. Aspects of the dsm production with high resolution images. International Archives of the Photogrammetry, Remote Sensing and Spatial Information Sciences 37(B4), pp. 1143-1146.
Montoya-Zegarra, J. A., Wegner, J.-D., Ladickỳ, L. and Schindler, K., 2015. Semantic segmentation of aerial images in urban areas with class-specific higher-order cliques. ISPRS Annals of the Photogrammetry, Remote Sensing and Spatial Information Sciences 2(3), pp. 127.

Poullis, C. and You, S., 2010. Delineation and geometric modeling of road networks. ISPRS Journal of Photogrammetry and Remote Sensing 65(2), pp. 165-181.

Rottensteiner, F., Sohn, G., Gerke, M. and Wegner, J. D., 2013. Isprs test project on urban classification and $3 \mathrm{~d}$ building reconstruction. Commission III-Photogrammetric Computer Vision and Image Analysis, Working Group III/4-3D Scene Analysis pp. $1-17$.

Sherrah, J., 2016. Fully convolutional networks for dense semantic labelling of high-resolution aerial imagery. arXiv preprint arXiv: 1606.02585.

Soergel, U., Cadario, E., Gross, H., Thiele, A. and Thoennessen, U., 2006. Bridge detection in multi-aspect high-resolution interferometric sar data. EUSAR 2006.

Stańczyk, U. and Jain, L. C., 2015. Feature selection for data and pattern recognition. Springer.

Türetken, E., Benmansour, F. and Fua, P., 2012. Automated reconstruction of tree structures using path classifiers and mixed integer programming. In: Computer Vision and Pattern Recognition (CVPR), 2012 IEEE Conference on, IEEE, pp. 566-573.

Ünsalan, C. and Sirmacek, B., 2012. Road network detection using probabilistic and graph theoretical methods. IEEE Transactions on Geoscience and Remote Sensing 50(11), pp. 44414453.

Valero, S., Chanussot, J., Benediktsson, J. A., Talbot, H. and Waske, B., 2010. Advanced directional mathematical morphology for the detection of the road network in very high resolution remote sensing images. Pattern Recognition Letters 31(10), pp. 1120-1127.

Varma, M. and Zisserman, A., 2005. A statistical approach to texture classification from single images. International Journal of Computer Vision 62(1-2), pp. 61-81.

Veksler, O., Boykov, Y. and Mehrani, P., 2010. Superpixels and supervoxels in an energy optimization framework. In: European conference on Computer vision, Springer, pp. 211-224.

Wang, W., Yang, N., Zhang, Y., Wang, F., Cao, T. and Eklund, P., 2016. A review of road extraction from remote sensing images. Journal of Traffic and Transportation Engineering (English Edition) 3(3), pp. 271-282.

Warnke, S., 2017. Variable Selection for Random Forest and Logistic Regression applied to Road Network Extraction from Aerial Images. Master thesis at Karlsruhe Institute of Technology, Germany.

Wegner, J. D., Montoya-Zegarra, J. A. and Schindler, K., 2015. Road networks as collections of minimum cost paths. ISPRS Journal of Photogrammetry and Remote Sensing 108, pp. 128 137.

Winn, J., Criminisi, A. and Minka, T., 2005. Object categorization by learned universal visual dictionary. In: Computer Vision, 2005. ICCV 2005. Tenth IEEE International Conference on, Vol. 2, IEEE, pp. 1800-1807. 

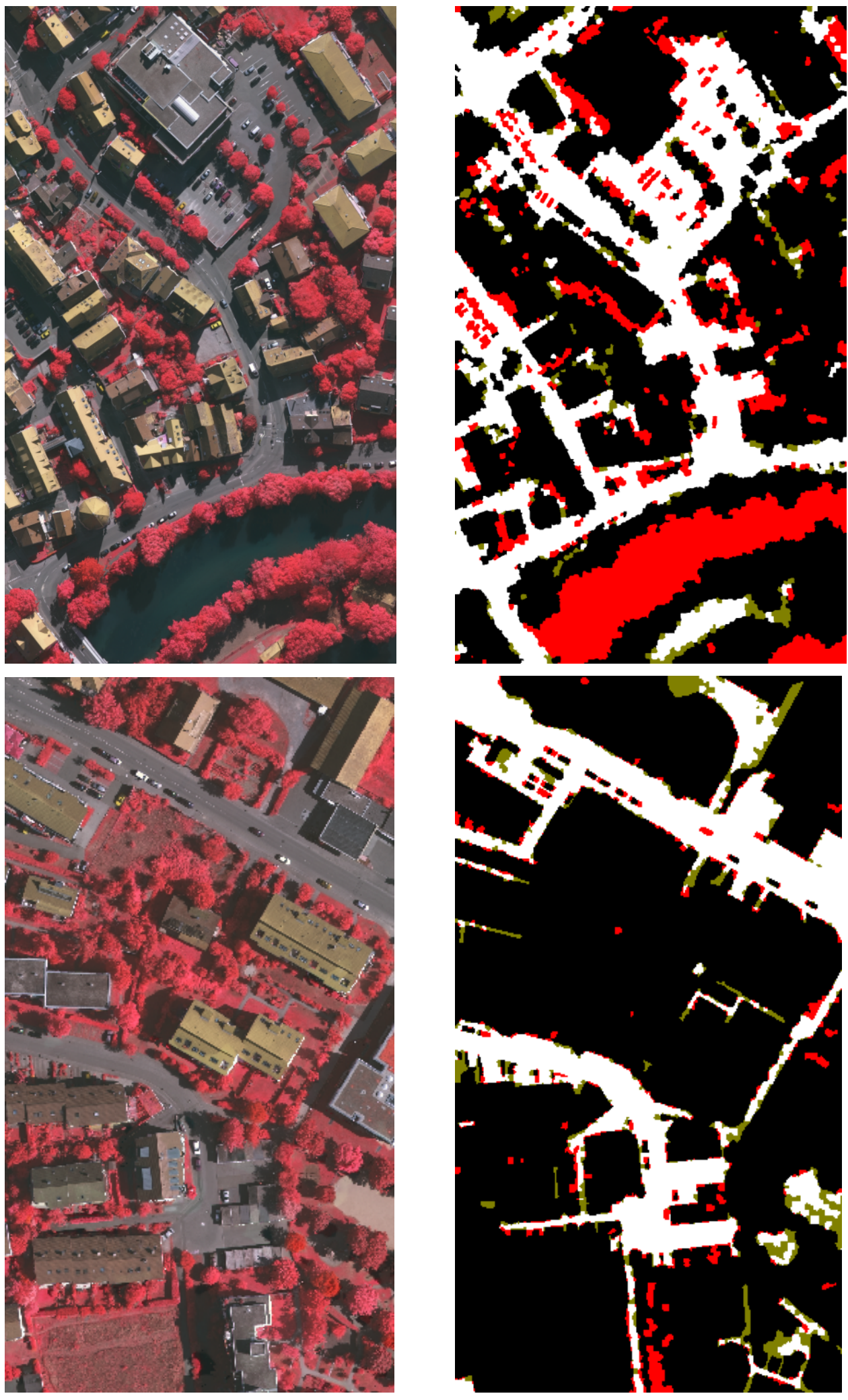

Figure 4: Results of classification: average patch, Random Forests classifier (below), challenging patch, Logistic Regression (above). On the left, the orthophoto and on the right, the classification result (white and black: true positives and negatives, dark-green and red: false positives and negatives, respectively). 